\title{
Prevalencia de enfermedades periodontales, factores de riesgo y necesidad de tratamiento en el personal de tropa masculino en Servicio Militar en Lima en el año 2000
}

Otero J, Proaño D. Prevalencia de enfermedades periodontales, factores de riesgo y necesidad de tratamiento en el personal de tropa masculino en Servicio Militar en Lima en el año 2000. Rev Estomatol Herediana 2005;15(1): $11-17$

\section{Juana Otero Purizaga ${ }^{1}$ Doris Proaño de Casalino²}

'Odontóloga asistente Policlínico Militar Rimac. 'Docente del Departamento Académico de Clínica Estomatológica. Facultad de Estomatología. Universidad Peruana Cayetano Heredia.

\section{Correspondencia}

Juana Otero Purizaga,

Jardines de Aramburú II E-10 A-101, Lima 34 - Perú. Telfs: 2417669-96391224.

E-mail: juanaotero@hotmail.com

Aceptado para publicación: 1 de marzo del 2005

\section{RESUMEN}

El propósito del presente estudio fue determinar la prevalencia de enfermedades periodontales, factores de riesgo y su consecuente necesidad de tratamiento en el personal masculino entre 17 y 21 años que ingresó en el año 2000 al servicio militar en Lima. El estudio fue transversal descriptivo. La muestra consistió en 263 sujetos elegidos por muestreo aleatorio sistemático. La condición periodontal y necesidad de tratamiento se midieron usando los índices siguientes: Índice periodontal comunitario de necesidad de tratamiento (IPCNT) y pérdida de fijación. Los factores de riesgo se obtuvieron usando el índice de higiene oral simplificado y mediante preguntas contenidas en la ficha de examen. El análisis de variables se hizo usando tablas de frecuencia y se usó la prueba chi cuadrado para la evaluación de la significancia diferencial. Los resultados mostraron que la placa bacteriana tiene una relación muy significativa $(\mathrm{P}=0,001)$ con el índice periodontal comunitario de necesidad de tratamiento. La prevalencia de los hallazgos fue como sigue: de cálculos dentarios fue de $77,4 \%$, de profundidad al sondaje (entre $4-5 \mathrm{~mm}$ ) fue de $22,4 \%$, hemorragia al sondaje de $0,4 \%$, pérdida de fijación entre $4-5 \mathrm{~mm}$ fue de $21,5 \%$ y $=6 \mathrm{~mm}$ fue de $1,1 \%$. Los sujetos en estudio necesitan educación en higiene bucal y raspado profesional. Esta investigación aporta datos respecto a la salud periodontal en la población adulta joven peruana.

Palabras clave: ENFERMEDADES PERIODONTALES. epidemiología / PERSONAL MILITAR.

Prevalence of periodontal diseases, risk factors, and treatment needs among male personnel on military service in Lima in the year 2000

ABSTRACT

The aim of the present study was to determine the prevalence of periodontal diseases, risk factors and consequent treatment needs among male personnel enrolled in the military service (17 to 21 years old) in Lima in the year 2000. This was a descriptive and transverse study. The sample consisted of 263 random systematically selected subjects. Periodontal status and treatment needs were measured using the Community periodontal index of treatment needs and attachment loss indexes; risk factors were assessed using the oral hygiene index (OHI) and via a questionnaire. Variables were analysed using frequency tables and the chi-square test. Results showed that dental plaque was significantly related to Community periodontal index of treatment needs $(\mathrm{P}=0,001)$. Prevalence of results were as follows: oral calculus was $77.4 \%$; probing pockets depth $(4-5 \mathrm{~mm}) 22.4 \%$; bleeding on probing $0.4 \%$, attachment loss $(4-5 \mathrm{~mm})$ was $21.5 \%$ and $=6 \mathrm{~mm}$ was $1.1 \%$. Study subjects needed oral hygiene education and professional scaling. This investigation provides data with respect to the periodontal health of the young Peruvian adult population.

Keywords: PERIDONTAL DISEASES. epidemiology / MILITARY PERSONNEL.

\section{Introducción}

Prevalencia es el número de casos existentes en una población en un punto específico en el tiempo $(1,2)$. Los datos epidemiológicos sobre caries dental han sido recopilados por décadas, mientras que las investigaciones periodontales son más recientes (3). El diagnóstico se basa principalmente en signos clínicos que incluyen: placa, cálculo, sangrado al sondaje, profundidad al sondaje (PS), pérdida de fijación (PF), recesión y pérdida dentaria, así como la evidencia radiográfica de pérdida ósea (4).

Las enfermedades periodontales (EEPP) y la caries dental son las enfermedades orales más prevalentes en el hombre (5) y también la causa primaria de pérdida de dientes permanentes en el hombre (5-7). Las EEPP han estado presentes durante toda la historia de la humanidad; ya que, cráneos antiguos de personas jóvenes, mostraron evidencias claras de pérdida de hueso periodontal (8). Ramfjord (9) hace más de cuatro décadas encontró una alta prevalencia de EEPP en jóvenes. En los más ancianos es la caries la razón principal de extracción, según estudios recientes $(10,11)$.

La clasificación de las enfermedades periodontales se basa en el concepto que las EEPP son el resultado de la interacción huésped-parásito (10-12). La severidad es variable dependiendo del tipo de placa bacteriana y la respuesta del huésped (2,3,8,10).

Actualmente se reconoce a EEPP como un factor de riesgo para la enfer 
medad cardiovascular y niños con bajo peso al nacer $(5,6,10)$.

Cutress et al. (13) en 1987, recomendaron que la distribución porcentual por sujetos, de acuerdo a las puntuaciones altas del índice comunitario de necesidades de tratamiento (IPCNT) pueden ser usadas como indicador de prevalencia en las evaluaciones de condición periodontal en la comunidad como también en el individuo. Este índice ha demostrado ser altamente sensible para estimar la prevalencia de profundidad al sondaje de $=4 \mathrm{~mm}$ y $=6 \mathrm{~mm}$, con $96 \%$ y $90 \%$ respectivamente (14); es por ello que el IPCNT es muy usado en las estimaciones de necesidades de tratamiento (NT) $(13,15)$; y en muchos casos también para hacer generalizaciones sobre EEPP $(16,17)$, además es el método usado y recomendado por la OMS para exámenes de población $(3,11,18)$; el IPCNT ha contribuido a formar el Banco Global de datos de Salud Oral de la OMS, además de tener aceptación universal $(11,19)$.

La pérdida de fijación (PF) es la distancia desde la unión cemento-esmalte (UCE) hasta el valor máximo de profundidad al sondaje, y representa la mejor medida clínica de severidad de la enfermedad, en términos de pérdida de soporte del diente $(14,20,21)$.

Los datos obtenidos en el Banco Global de datos en salud oral de la OMS están desactualizados para algunos países y para otros no existen (3). Muy pocos estudios de prevalencia se han hecho en América del Sur (22). Gjermo et al. (23) en 1984 realizaron un estudio en jóvenes brasileños, donde la PF fue diagnosticada en $28 \%$ de los sujetos, $2,6 \%$ fueron diagnosticados con periodontitis agresiva y 25,4\% con periodontitis crónica; a su vez, Albandar et al. (24) en 1991 examinaron a 222 adolescentes de un colegio privado de Sao Paulo- Brasil durante tres años, encontrando $1,3 \%$ y $1,8 \%$ de periodontitis agresiva en los sujetos de 13 a 16 años respectivamente. Tinoco et al. (25) en 1997 examinaron a sujetos jóvenes brasileños de 12 a 19 años y encontraron que la prevalencia de periodontitis agresiva fue de $0,3 \%$, similar porcentaje de prevalencia fue encontrado por López et al. (26) datos de muchas partes del mundo muestran que la prevalencia de periodontitis severa está en un promedio de 7 a 15\% en casi todas las poblaciones, independiente de su desarrollo económico, higiene oral o la eficacia de los cuidados dentales $(2,3,8,10,27)$.

El cálculo dental es un factor importante para la severidad de la enfermedad periodontal $(9,21,28,29)$. Se ha reportado que la formación del cálculo tiene patrones diferentes en poblaciones que practican una higiene oral regular y con acceso a cuidados profesionales, con respecto a los no atendidos; la formación de cálculos es limitada en poblaciones con acceso a cuidados dentales $(30,31)$.

El inicio y el progreso de las infecciones periodontales están modificadas por condiciones locales, sistémicas y genéticas denominadas factores de riesgo $(6,21,32)$.

El consumo de cigarrillos se asocia fuertemente con el incremento de prevalencia y severidad de EEPP, se sospecha que juega un rol importante en la patogénesis de las EEPP (2,6,8,21,33-38), y puede afectar el resultado de las terapias quirúrgicas (39). El cepillo dental es el dispositivo más usado para la higiene oral con el propósito de remover la placa dental; cuando se utiliza correctamente y el paciente es instruido, se ha observado una reducción de aproximadamente el 30\% de los niveles de placa comparada con la higiene oral ordinaria (40). Abegg (41) estudió los hábitos orales de la población adulta joven del Brasil y encontró que el 67,5\% usaba hilo dental y 54,6\% palillos de dientes.

\section{Materiales y método}

La población estuvo representada por 5.016 individuos de sexo masculino entre 17 y 21 años de edad que ingresaron al servicio militar en el año 2000, en la ciudad de Lima. Se usó una muestra representativa de 263 sujetos.

La necesidad de tratamiento se midió usando el índice periodontal comunitario y de necesidad de tratamiento (IPCNT) $(1,4,13-15,19,42,43)$; la condición periodontal se midió con el índice de pérdida de fijación (PF) (2-4,43); la higiene oral con el índice de Greene y Vermillion simplificado $(1,4,19,44)$; el estado nutricional se determinó utilizando el índice de masa corporal (45); la edad se registró en años; la procedencia se registró según regiones geográficas, considerando lugar de procedencia la última localidad donde residió el sujeto como mínimo cinco años; también se registraron los hábitos de consumo de tabaco y de higiene oral; así como, los artículos de higiene.

La calibración intraexaminador $(5,14,46,47)$ se hizo a través del examen de cinco pacientes tomados al azar con un intervalo de 30 minutos entre el examen inicial y el siguiente. La calibración interexaminador $(5,14,47)$ se realizó a través del examen de cinco pacientes tomados al azar, con un intervalo de 15 minutos entre pacientes. Se obtuvo un Kappa de 1,0 para el IPCNT; 0,89 para el índice de pérdida de fijación y 0,8 para el índice de Greene y Vermillion.

Se realizó análisis univariado de todas las variables y covariables; además, se realizó análisis bivariado para establecer la relación entre las variables a través de la prueba de chi cuadrado.

\section{Resultados}

$\mathrm{Al}$ análisis univariado se obtuvo: La edad promedio de los participantes fue de 19 años (35,1\%), seguido de cerca $(33,2 \%)$ por los de 18 años (Tabla 1$)$.

La gran mayoría de individuos (57,4\%) procedía de la costa, un 31,3\% de la sierra y el 11,3\% de la selva; con un tiempo de residencia en la región de procedencia de 5 años.

El 63,4\% fumaba, de ellos el 61,9\% fumaba entre 1 a 10 cigarrillos (Tabla 2).

El 79,2\% mostró un estado nutricional normal, el $12,5 \%$ bajo peso y el 8,3\% sobrepeso.

La mayoría (65,3\%) manifestó cepillarse 2 veces al día y el 0,8\% ninguna vez (Tabla 3).

El 3,4\% si usa hilo dental, frente al $96,6 \%$ que no usa; de estos últimos, el $1,1 \%$ usa hilo de coser; palillos de dientes $(1,1 \%)$, palitos de fósforo $(0,4 \%)$, palitos $(0,4 \%)$ y sal $(0,4 \%)$. 
El índice de higiene oral fue de 1,3 a 3,0 (regular) en 54,7\% y de 3,1 a 6,0 (malo) en $40,4 \%$ frente a un $4,9 \%$ de 0 a 1,2 (bueno).

El IPCNT encontrado, en su mayoría $(77,4 \%)$ fue de 2 ; es decir, presentan cálculos a la exploración con la sonda (Tabla 4).

La pérdida de fijación fue 0 con
$77,4 \%$, es decir una pérdida de fijación entre 0 a 3 mm (Tabla 5). El 21,5\% con pérdida de fijación entre 4 y 5 mm y sólo tres personas $(1,1 \%)$ presentaron pérdida de fijación entre 6 y 8 mm

Del análisis bivariado se obtuvo: La Tabla 6 muestra la relación IHO-s e IPCNT. Se encontró relación muy significativa entre IHO-s e IPCNT $(\mathrm{P}=0,001)$.

\section{Discusión}

El propósito del estudio fue establecer la prevalencia de enfermedades periodontales, factores de riesgo y su consecuente necesidad de tratamiento en el personal masculino que presta servicio militar de Lima.

Para estimar la NT en una población
Tabla 1. Distribución de la variable edad en la muestra de individuos de sexo masculino entre 17 y 21 años de edad que ingresaron al servicio militar en el año 2000, en la ciudad de Lima.

\begin{tabular}{llr}
\hline Edad & Frecuencia & $\%$ \\
\hline 17 & 20 & 7,5 \\
18 & 88 & 33,2 \\
19 & 93 & 35,1 \\
20 & 52 & 19,6 \\
21 & 12 & 4,5 \\
\hline Total & 265 & 100 \\
\hline
\end{tabular}

Tabla 4. Distribución de la variable IPCNT en la muestra de individuos de sexo masculino entre 17 y 21 años de edad que ingresaron a servicio militar en el año 2000, en la ciudad de Lima.

\begin{tabular}{llc}
\hline IPCNT & Frecuencia & $\%$ \\
\hline 0 & 0 & 0 \\
1 & 1 & 0,4 \\
2 & 205 & 77,3 \\
3 & 59 & 22,4 \\
4 & 0 & 0 \\
X & 0 & 0 \\
9 & 0 & 0 \\
\hline Total & 265 & 100 \\
\hline
\end{tabular}

Tabla 6. Análisis bivariado de la relación IPCNT e higiene oral (IHO-s) en la muestra de individuos de sexo masculino entre 17 y 21 años de edad que ingresaron al servicio militar en el año 2000, en la ciudad de Lima.

\begin{tabular}{lllll}
\hline IPCNT & $\begin{array}{l}\text { IHO-s } \\
\text { Bueno }\end{array}$ & Regular & Malo & Total \\
\hline 0 & 0 & 0 & 0 & 0 \\
1 & 1 & 0 & 0 & 1 \\
2 & 10 & 112 & 83 & 205 \\
3 & 2 & 33 & 24 & 59 \\
4 & 0 & 0 & 0 & 0 \\
X & 0 & 0 & 0 & 0 \\
9 & 0 & 0 & 0 & 0 \\
\hline Total & 13 & 145 & 107 & 265 \\
\hline
\end{tabular}

Chi cuadrado : 19,679 • Grados de libertad: 4 • Valor P: 0,001
Tabla 2. Distribución de la variable número de cigarrillos diarios fumados en la muestra de individuos de sexo masculino entre 17 y 21 años de edad que ingresaron al servicio militar en el año 2000, en la ciudad de Lima.

\begin{tabular}{llr}
\hline $\begin{array}{l}\text { Número de } \\
\text { cigarrillos }\end{array}$ & Frecuencia & $\%$ \\
\hline 1 a 10 & 164 & $\begin{array}{r}61,9 \\
1,5\end{array}$ \\
Más de 10 & 4 & 63,4 \\
\hline Total & 168 & \\
\hline
\end{tabular}

Tabla 5. Distribución de la variable pérdida de fijación en la muestra de individuos de sexo masculino entre 17 y 21 años de edad que ingresaron al servicio militar en el año 2000 en la ciudad de Lima.

\begin{tabular}{llc}
\hline Pérdida de fijación & Frecuencia & $\%$ \\
\hline 0 & 205 & 77,4 \\
1 & 57 & 21,5 \\
2 & 3 & 1,1 \\
3 & 0 & 0 \\
4 & 0 & 0 \\
$X$ & 0 & 0 \\
9 & 0 & 0 \\
\hline Total & 265 & 100 \\
\hline
\end{tabular}

Tabla 3. Distribución de la variable frecuencia de cepillado en la muestra de individuos de sexo masculino entre 17 y 21 años de edad que ingresaron al Servicio Militar en el año 2000 , en la ciudad de Lima.

\begin{tabular}{llr}
\hline $\begin{array}{ll}\text { Frecuencia de } \\
\text { cepillado }\end{array}$ & Frecuencia & \multicolumn{1}{c}{$\%$} \\
\hline Ninguna & 2 & 0,8 \\
$1 \mathrm{v}$ & 51 & 19,2 \\
$2 \mathrm{v}$ & 173 & 65,3 \\
$3 \mathrm{v}$ & 39 & 14,7 \\
\hline Total & 265 & 100 \\
\hline
\end{tabular}

se debe tener en cuenta el cálculo dental y la profundidad al sondaje (PS) y si se desea describir los niveles de EEPP se deben utilizar parámetros que incluyan: el sangrado gingival, cálculo, PS y PF (2,14); por ello, se utilizó el IPCNT y la PF, que contienen los parámetros descritos anteriormente. La prevalencia de EEPP está basada normalmente, en el valor máximo de PS y la PF por sujeto; asimismo, la severidad de las EEPP puede ser reportada como un valor promedio de la PF (14). Se encontró que 22,4\% tuvieron una PS de $=4 \mathrm{~mm}$, una PF entre 4-5 mm de $21,5 \%$ y de $=6 \mathrm{~mm}$ de $1,1 \%$.

Todos los sujetos examinados mostraron presencia de por lo menos un signo de enfermedad periodontal, al igual que lo informado por Ramfjord (9) respecto del estado periodontal en adolescentes de 11 a 17 años en Bombay (India). Igualmente en África son raras las personas con periodonto sano (IPCNT O) (18), mientras que en los EEUU un $65,5 \%$ no tienen periodontitis (7). Todo esto confirmaría que las enfermedades periodontales son las más prevalentes en el hombre (5). El mayor porcentaje de individuos periodónticamente sanos en 
los EEUU se debe probablemente al mayor acceso a cuidados dentales, políticas nacionales de salud oral y mejor nivel educativo (7).

El sangrado al sondaje en la población adulta de EEUU es de 50,3\% en por lo menos un diente y fue más extensa en hombres que en mujeres $(7,29)$, esto contrasta con nuestro estudio donde todos los sujetos son varones y el porcentaje de sangrado al sondaje es de sólo $0,4 \%$; porcentajes similares han sido reportados en poblaciones africanas (18).

El valor más frecuente de IPCNT 2, nos pone de manifiesto que esta población en su gran mayoría presenta cálculos dentarios (77,3\%); en datos obtenidos en el III estudio nacional de salud y nutrición en EEUU, se encontró también un alto porcentaje (92\%) de personas adultas con uno o más dientes con cálculo dental en todos los grupos de edades y más alta en hombres que en mujeres (7). En una realidad totalmente opuesta, donde no se cuenta con acceso a cuidados dentales, donde existe pobreza extrema, escasez de posibilidades educativas, baja expectativa de vida; todas éstas características del continente africano, cuentan con una alta prevalencia de cálculos dentales, especialmente para el grupo de 15-19 años y de 35-44 años, de acuerdo a los datos obtenidos con el IPCNT (18); debiendo tener presente que en poblaciones que tienen regular acceso a cuidados profesionales los cálculos se incrementan con la edad hasta los 30 años como máximo $(7,28)$ y según amplia evidencia, éstos son un factor de riesgo importante para la severidad de la enfermedad periodontal $(9,21,28,29)$. Los niveles de cálculo, su formación y localización son afectadas por hábitos de higiene oral, acceso a cuidados profesionales, dieta, edad, origen étnico, tiempo desde la última limpieza dental, enfermedades sistémicas y el uso de medicamentos (30). Así mismo, los cálculos dentales son una determinante importante en el inicio de la recesión gingival, se observa recesión gingival avanzada en jóvenes (20-34 años) de poblaciones que no tienen acceso a cuidados dentales profilácticos (31).
El 64\% de personas adultas de los EEUU tienen uno o más dientes con PS $=3 \mathrm{~mm}$ y aproximadamente $23 \%$ tienen PS =4 mm; ésta última fue significativamente alta en varones que en mujeres (7); en nuestro estudio la PS entre 4 y $5 \mathrm{~mm}$ fue de $22,4 \%$, no se presentó ningún sujeto con PS $=6 \mathrm{~mm}$; estos hallazgos presentan cierta similitud con los datos del continente africano, hallados también con el IPCNT; en los que la PS $=4 \mathrm{~mm}$ son relativamente frecuentes y la PS $=6 \mathrm{~mm}$ son infrecuentes (18).

Se ha sugerido $4 \mathrm{~mm}$ de PF como punto de corte para estudios epidemiológicos, ya que ésta representa una condición seria, porque sólo por encima de este nivel la función del diente puede verse comprometida y el riesgo de pérdida dentaria se incrementa (3). En un estudio descriptivo realizado en adolescentes Brasileños (23) (15 años) de una población con bajo nivel socioeconómico; se usaron radiografías bite-wing para evaluar las EEPP, donde se usó como criterio de punto de corte para $\mathrm{PF}>2 \mathrm{~mm}$; se encontró que el 28\% de sujetos tenían PF y 2,6\% tuvieron periodontitis agresiva (pérdida de hueso vertical en $=3$ primeras molares. Tinoco et al. (25) usando una muestra más grande (7.843 niños y adolescentes entre 12 y 19 años Brasileños), halló un $0,3 \%$ de prevalencia para periodontitis agresiva; la razón probable para este porcentaje menor con respecto a los otros estudios, sería que se seleccionaron sujetos con PS $=5 \mathrm{~mm}$, y sólo a estas se les tomaron radiografías para confirmar la pérdida ósea (>2 mm). Utilizando el mismo procedimientos y parámetros López et al.(26) realizaron un estudio transversal en 2500 sujetos de 15 a 16 años en Santiago de Chile, encontrando similar porcentaje (0,32\%) de prevalencia de periodontitis agresiva. La disparidad de porcentajes encontrados podría deberse a la diferencia de los parámetros utilizados. Todos los estudios demuestran una alta prevalencia de periodontitis agresiva en América del Sur, comparada con los países industrializados (0,1 a 0,5\% en Europa del Este; 0,4 a 0,8 \% en Norteamérica) (22).

López et al. (48) condujeron un es- tudio transversal en estudiantes Chilenos de 12 a 21 años, encontrando que sólo el $4,5 \%$ tenía una $\mathrm{PF}=3 \mathrm{~mm}$ entre los 15 a 17 años y 8,2\% para los de 18 a 21 años; estos investigadores concluyeron que el incremento de la edad, una higiene oral pobre y el bajo nivel socioeconómico jueguen roles en la ocurrencia de PF entre los adolescentes chilenos; mientras que en nuestro estudio no hubo relación entre edad y condición periodontal o PF, esto probablemente se deba al tamaño muestral y al grupo etáreo estudiado. En la población Norteamérica se ha demostrado la relación entre prevalencia y extensión de periodontitis con la edad $(10,47)$, de igual forma ocurre con la población africana (18).

Albandar et al. (49) al estudiar la prevalencia de periodontitis agresiva en sujetos de 12 a 25 años en Uganda; encontraron que existía relación entre el incremento de la enfermedad y la edad; así, el porcentaje de periodontitis agresiva aumentó de $27 \%$ en los estudiantes de 12 a 16 años a $35 \%$ en los de 20 a 25 años. Se debe tener en cuenta que este fue un estudio transversal y que la muestra fue relativamente pequeña.

Poblaciones de diferentes regiones poseen características y diferencias no sólo en sus tradiciones sino también en: su etnia, grado de heterogeneidad genética, patrones de segmentación, diferencias demográficas y socioeconómicas que pueden modificar o alterar la ocurrencia, extensión y severidad de las EEPP (38)

En Chile, cerca del 40\% de jóvenes entre los 13 a 15 años fuman habitualmente. El área andina presenta la prevalencia de tabaquismo actual más alta; seguido por los EEUU, Costa Rica, México, el Caribe latino y el Caribe angloparlante (50). Los datos proporcionados por la National Health Interview Survey de 1997 de personas de 18 años a más, muestra que la preponderancia actual general sobre el fumar en adultos hispanos es de 20,4\%, comparada con un 16,9\% entre los descendientes de Asia y las Islas del Pacífico, 25,3\% entre los blancos, 26,7\% para africano-americanos y 34,1\% entre los indios norteamericanos y nativos de 


\section{Alaska (51).}

En el 2001 el consumo en el Perú cayó en 8,2\% frente al año anterior; se sugiere que la causa sea el bajo poder adquisitivo de la población (52). En un análisis de la situación del Perú hecho el 2002, se encontró que el 50\% de adolescentes y adultos jóvenes de 17 a 19 años consumen tabaco (53). La prevalencia de tabaquismo en jóvenes de 13 a 15 años en Lima fue $23,4 \%$, en Trujillo 18,7\% y en Tarapoto 18,7 \%, según datos del 2002 (50).

En un estudio realizado por Hashim et al.(54) en sujetos entre 15 a 26 años de Nueva Zelanda, de tipo cohorte prospectivo se investigó el rol de la exposición crónica de fumar cigarrillos como un factor de riesgo para la prevalencia y extensión de PF. Encontraron que la $\mathrm{PF}=4 \mathrm{~mm}$ ocurrió en promedio $33,6 \%$ en las edades de 15, 18, 21 y 26 años; llegando a la conclusión que la exposición crónica de fumar es un fuerte predictor para la prevalencia de EEPP en adultos jóvenes. Asimismo un reporte sobre los efectos de fumar tabaco en adolescentes Indios Navajos entre los 14 a 19 años (37). En el presente trabajo se encontró que, independientemente de la región de procedencia, la mayoría fumaba; de ellos, el 61,9\% fumaba de 1 a 10 cigarrillos diarios, en la muestra del estudio, los sujetos fumaban 5,4 cigarrillos por día durante tres años, que es una exposición relativamente baja comparada con otras poblaciones reportadas en la literatura y ésta puede ser la razón de la escasa asociación reportada (55); no hubo relación estadística entre fumar e IPCNT o PF, probablemente debido al tamaño muestral y la baja exposición.

Se ha reportado que a mayor número de cigarrillos mayor puntuación de IPCNT y pérdida de fijación, lo cual sugeriría que el hábito de fumar juegue un rol preponderante en la prevalencia e intensidad de las EEPP (2,6,8,35-38).

La remoción mecánica de placa es un aspecto básico para la reducción de organismos patógenos y una buena salud de los tejidos periodontales, la eficacia del cepillado dental está correlacionado con la frecuencia y uso de pasta dental (40). En nuestro estudio el $65,3 \%$ de sujetos se cepilla dos veces por día, esto ocurre porque a pesar de provenir de diferentes regiones y estratos, en el servicio militar se les indica y supervisa que se aseen los dientes por lo menos dos veces por día; sin embargo, la falta de información sobre higiene dental origina que casi el 96,6\% no usen hilo dental y el 3,4\% utilicen sustitutos domésticos como: hilo de coser, palitos de dientes o fósforos y algunos sal común con la idea de higienizar sus dientes. Petersilka et al. (40) mencionaron que la limpieza con cepillo interdental se extiende hasta $2,5 \mathrm{~mm}$ debajo del margen gingival, mientras que el hilo dental puede penetrar hasta 3,5 $\mathrm{mm}$. Debemos recordar que el uso de palitos de dientes es considerado un factor de riesgo para gingivitis (18), sin embargo el uso de palitos de madera, de hilo dental, cepillos interdentales manuales y eléctricos ha aumentado en la población (10\%), ya que múltiples publicaciones han reportado que las áreas interdentales no pueden ser limpiadas solo por cepillado dental (40). Abegg (41) estudió los hábitos orales de trabajadores del Brasil y concluyó que la mayoría se cepilla con una frecuencia de tres veces por día; menos de $1 \%$ reportó hacerlo una vez al día. El 67,5\% manifestaron haber usado hilo dental y $54,6 \%$ utilizaron palitos de dientes y este hábito aumenta con el incremento de la edad. En el continente africano el uso de palitos de dientes es muy común como procedimiento de limpieza dental, siendo más utilizado que el cepillado dental para la remoción de placa (18).

Axelsson et al.(56) en un estudio longitudinal de 15 años mostraron que niveles adecuados de higiene oral mantenidos profesionalmente, pueden ser efectivos en la prevención de la recurrencia de EEPP (11) en niños como en adultos.

La relación IHO-s y IPCNT encontrada en nuestro estudio es muy significativa ( $\mathrm{P}=0,001)$, lo cual reforzaría lo ampliamente documentado que la placa bacteriana es un factor de riesgo para la aparición y progreso de las EEPP (2,6$8,11,16,38)$. Debemos tener presente que en cuanto a enfermedad periodontal, los pacientes no son iguales en el riesgo de desarrollar la enfermedad y las superficies de los dientes son afectadas de manera variable $(10,57)$.

La puntuación mayoritaria de IPCNT 2 encontrado en nuestro estudio indica que esta población necesita mejorar su higiene oral y realizar el raspado profesional correspondiente; ya que, estos son tratamientos efectivos en el manejo de pacientes con gingivitis y periodontitis leve y moderada (10), según los parámetros de tratamiento de la AAP (5860).

La necesidad de determinar la prevalencia de estas enfermedades es importante para establecer las necesidades de tratamiento para grupos poblacionales particulares (61), además este estudio ha puesto de manifiesto la urgente necesidad de realizar estrategias para educar a estos individuos sobre salud oral y la participación familiar, comunitaria e individual son requisitos esenciales en los programas preventivos de salud periodontal. La finalidad debe ser la de lograr una mayor conciencia individual acerca de la importancia de los tejidos gingivales sanos para poder conservar una dentición sana y funcional durante toda la vida $(62,63)$.

\section{Conclusiones}

- La prevalencia de cálculos dentarios es de $77,4 \%$, de profundidad al sondaje entre 4 y $5 \mathrm{~mm}$ fue de 22,4\%, hemorragia al sondaje de $0,4 \%$, PF entre 4 y $5 \mathrm{~mm}$ de $21,5 \%$ y $=6 \mathrm{~mm}$ de $1,1 \%$.

- La prevalencia de condiciones de IPCNT está muy asociada a la presencia de placa bacteriana $(\mathrm{P}=0,001)$.

- Los sujetos en estudio requieren educación en higiene bucal y raspado profesional de sus dientes.

- No se observa relación entre condición periodontal y necesidad de tratamiento según estado nutricional, edad, procedencia, frecuencia de cepillado, hilo dental y otros hábitos de higiene oral.

- Si bien no se observó relación entre condición periodontal y necesidad de tratamiento según hábito de fu 
mar, debe tenerse en cuenta la relación causa-efecto de éste hábito y la progresión y severidad de las EEPP.

\section{Referencias bibliográficas}

1. Carranza FJr, Perry DA. Manual de Periodontología Clínica. 3a ed. México: Nueva Editorial Interamericana S.A.; 1986.

2. Position paper: epidemiology of periodontal diseases. American Academy of Periodontology. J Periodontol. 1996;67(9):935-45.

3. Baehni PC, Bourgeois DM. Epidemiology of periodontal health and disease. En: Lang NP, Attström R, Loë EH. Proceedings of the European Workshop on mechanical plaque control. 1a ed. Berlin: Quintessence Books 1998: 19-34.

4. Lang NP. Commonly used indices to assess oral hygiene and gingival and periodontol health and diseases. En: Lang NP, Attström R, Loë EH. Proceedings of the European Workshop on mechanical plaque control.1a ed. Berlin: Quintessence Books 1998: 50-71.

5. Albandar JM, Brunelle JA, Kingman A. Destructive periodontal disease in adults 30 years of age and older in the United States, 1988-1994. J Periodontol. 1999;70(1):13-29.

6. Genco RJ. Current view of risk factors for periodontal diseases. J Periodontol. 1996;67(10 Suppl): 1041-9.

7. Albandar JM. Periodontal diseases in North America. Periodontol 2000. 2002;29:31-69.

8. Burt BA, Eklund SA. Dentistry, Dental Practice and the community. 4a ed. México: Editorial W.B Saunders Company; 1992: 113-128.

9. Ramfjord SP. The periodontal status of boys 11 to 17 years old in Bombay, India. J Periodontol. 1961; 32:237-248.

10. Greenstein G, Lamster I. Changing periodontal paradigms: Therapeutic implication. Int $\mathrm{J}$ Periodontics Restorative Dent. 2000;20(4):336-57.

11. Gjermo P, Rösing CK, Susin C, Opperman R. Periodontal diseases in Central and South America. Periodontol 2000. 2002;29:70-78.
12. Ranney RR. Classification of periodontal diseases. Periodontol 2000. 1993;2:13-25.

13. Cutress TW, Ainamo J, Sardo-lnfirri $\mathrm{J}$. The community periodontal index of treatment needs (CPITN) procedure for population groups and individuals. Int Dent J. 1987;37(4):222-33.

14. Kingman A, Albandar JM. Methodological aspects of epidemiological studies of periodontal diseases. Periodontol 2000. 2002; 29:11-30.

15. Ainamo J, Ainamo A. Validity and relevance of the criteria of the CPITN. Int Dent J. 1994;44(5 Suppl 1):527-32.

16. Lewis JM, Morgan MV, Wright FA. The validity of the CPITN scoring and presentation method for measuring periodontal conditions. J Clin Periodontol. 1994;21(1):1-6.

17. Pilot T, Miyazaki H. Global results: 15 years of CPITN epidemiology. Int Dent J. 1994;44(5 Suppl 1):553-60.

18. Baelum V, Scheutz F. Periodontal diseases in Africa. Periodontol 2000. 2002; 29:79-103.

19. Burt BA, Eklund SA. Dentistry, Dental Practice and the Community.4a ed. México: Editorial W.B. Saunders Company; 1992: 64-68

20. Holmgren CJ. CPITN-Interpretations and limitations. Int Dent J 1994; 44(5 Suppl 1):533-546.

21. Pihsltrom BL. Periodontal risk assessment, diagnosis and treatment planning. Periodontol 2000. 2001; 25:37-58.

22. Albandar JM, Tinoco EM. Global epidemiology of periodontal diseases in children and young persons. Periodontol 2000. 2002; 29:153-176.

23. Gjermo P, Bellini HT, Pereira Santos V, Martins JG, Ferracyoli JR. Prevalence of bone loss in a group of Brazilian teenagers assessed on bite-wing radiographs. J Clin Periodontol 1984; 11(2):104-113.

24. Albandar JM, Buischi YA and Barbosa MF. Destructive forms of periodontal disease in adolescents. A 3-year longitudinal study. J periodontol 1991; 62(6):370-6.

25. Tinoco EMB, Beldi MI, Loureiro CA, et al. Localized juvenile periodontitis and Actinobacillus actinomycetemcomitans in a Brazilian population. Eur J Oral Sci 1997; 105(1):9-14.

26. López NJ, Ríos V, Pareja MA, Fernández O. Prevalence of juvenile periodontitis in Chile. J Clin Periodontol 1991; 18(7):529-533.

27. Griffiths GS, Wilton JM, Curtis MA, et al. Detection of high risk groups and individuals for periodontal diseases. Clinical assessment of the periodontium. J Clin Periodontol 1988; 15(7):403-10.

28. Löe H, Ånerud A, Boysen H. The natural history of periodontal disease in man: prevalence, severity and extend of gingival recession. $\mathrm{J}$ Periodontol 1992; 63(6):489-95.

29. Albandar JM, Kingman A. Gingival recession, gingival bleeding, and dental calculus in adults 30 years of age and older in the United States, 1988-1994. J Periodontol 1999; 70(1):30-43.

30. White DJ. Dental calculus: recent insights into occurrence, formation, prevention, removal and oral health effects of supragingival and subgingival deposit. Eur J Oral Sci 1997; 105(5 Pt 2):508-522.

31. Van Palenstein Helderman WH, Lembariti BS, van der Weijden GA, van'T Hof MA: Gingival recession and its association with calculus in subjects deprived of prophylactic dental care. J Clin Periodontol 1998; 25(2):106-11.

32. Michalowicz BS, Aeppli D, Virag JG, et al. Periodontal findings in adult twins. J Periodontol 1991; 62(5):293299.

33. Consensus report: Chronic periodontitis. Ann Periodontol 1999; 4:38.

34. Consensus report: Aggresive periodontitis. Ann Periodontol 1999; 4:53.

35. Preber H, Bergström J, Linder LE. Occurrence of periopathogens in smoker and non-smoker patients. J Clin Períodontol 1992; 19(9 Pt 1):667- 
671.

36. Gelskey SC. Cigarette smoking and periodontitis: methodology to assess the strength of evidence in support of the causal association. Community Dent Oral Epidemiol 1999; 27(1):16-24.

37. Wolfe MD, Carlos JP. Oral health effects of smokeless tobacco use in Navajo Indian adolescents. Community Dent Oral Epidemiol 1987; 15(4):230-35.

38. Albandar JM. Global risk factors and risk indicators for periodontal diseases. Periodontol 2000 2002; 29:177-206.

39. Preber H, Bergstrom J. Effect of cigarette smoking on periodontal healing following surgical therapy. J Clin Periodontol 1990; 17: 324-328. Abstract.

40. Petersilka GJ, Ehmke B, Flemmig TF. Antimicrobial effects of mechanical debridement. Periodontol 2000 2002; 28:56-71.

41. Abegg C. Oral hygiene habits among Brazilian adults in an urban area of Southern Brazil. Revista Saude Pública 1997; 31:586-593.

42. Ainamo J, Barmes D, Beagrie G, Cutress T, Martin J, Sardo-Infirri J. Development of the World Health Organization (WHO) Community periodontal index of treatment needs (CPITN). Int Dent J 1982; 32(3): 281291.

43. OMS. Encuestas de Salud Bucodental. Métodos Básicos.4a ed. Ginebra; 1997.
44. Greene JC, Vermillion JR. The simplified oral hygiene index. JADA 1964; 68:25-31.

45. Sistema de información para la vigilancia alimentaria y nacional del Perú. Propuesta de procedimientos para la recolección de información. 02-12-2000.

46. Mullally $\mathrm{BH}$ and Linden GJ. Comparative reproducibility of proximal probing depth using electronic pressure-controlled and hand probing. J Clin Periodontol 1994; 21:284-288.

47. Reddy MS, Palcanis KG, Geurs NC. A comparison of manual and controlled-force attachment-level measurements. J Clin Periodontol 1997; 24:920-926.

48. López R, Fernández O, Jara G, Baelum V. Epidemiology of clinical attachment loss in adolescents. J Periodontol 2001; 72:1666-1672.

49. Albandar JM, Muranga MB, Rams TE. Prevalence of aggressive periodontitis in school attendees in Uganda. J Clin Periodontol 2002; 29:823-831.

50. OPS. OMS. Encuesta Mundial sobre tabaquismo en la juventud: Resultados en las Américas-2002.

51. Center of disease control and prevention. Cigarette smoking among adults - Unites States, 1997.MMWR.48: 993-6.

52. Diario el Comercio. "El mercado de los cigarrillos”. 28-04-2002.

53. OPS. Análisis de la situación Perú. Lima, Setiembre 2002.
54. Hashim R. Thomson WM, Pack AR. Smoking in adolescence as a predictor of early loss of periodontal attachment. Community Dent Oral Epidemiol 2001; 29:130-135.

55. Albandar JM, Rams TE. Risk factors for periodontitis in children and young persons. Periodontol 2000 2002; 29:207-222.

56. Axelsson P, Lindhe J, Nystrom B. On the prevention of caries and periodontal disease. Results of a 15year longitudinal study in adults. J Clin Periodontol 1991; 18:182-189.

57. Grbic JT, Lamster IB, Celenti RS and Fine JB. Risk indicators for future clinical attachment loss in adult periodontitis. Patient variables. J Periodontol 1991; 62:322-329.

58. Armitage GC. Development of a classification system for periodontal diseases and condition. Ann Periodontol 1999; 4:1-6.

59. Armitage GC. Periodontal diseases: diagnosis. Ann Periodontol 1996; 1:37-215.

60. AAP. Parameters of care. Supplement. J Periodontol 2000; 71:851-855.

61. Melvin WL, Sandifer JB and Gray JL. The prevalence and sex ratio of juvenile periodontitis in a young racially mixed population. J Periodontol 1991; 62:330-334.

62. Croxson LJ, Purdell-Lewis D. Periodontal health: CPITN as a promotional strategy. Int Dent J 